\title{
Topology and Content Co-Alignment Graph Convolutional Learning
}

\author{
Min Shi, Student Member, IEEE, Yufei Tang, Member, IEEE, and Xingquan Zhu, Senior Member, IEEE
}

\begin{abstract}
In traditional Graph Neural Networks (GNN), graph convolutional learning is carried out through topologydriven recursive node content aggregation for network representation learning. In reality, network topology and node content are not always consistent because of irrelevant or missing links between nodes. A pure topology-driven feature aggregation approach between unaligned neighborhoods deteriorates learning for nodes with poor structure-content consistency, and incorrect messages could propagate over the whole network as a result. In this paper, we advocate co-alignment graph convolutional learning (CoGL), by aligning the topology and content networks to maximize consistency. Our theme is to force the topology network to respect underlying content network while simultaneously optimizing the content network to respect the topology for optimized representation learning. Given a network, CoGL first reconstructs a content network from node features then co-aligns the content network and the original network though a unified optimization goal with (1) minimized content loss, (2) minimized classification loss, and (3) minimized adversarial loss. Experiments on six benchmarks demonstrate that CoGL significantly outperforms existing state-of-the-art GNN models.
\end{abstract}

Index Terms-Graph convolutional learning, graph mining, network embedding, network representation learning, neural networks

\section{INTRODUCTION}

Recent years have witnessed a significant growth of Graph Neural Networks (GNN) in solving domain specific tasks such as social network mining [1] and image recognition [2], etc. This is mainly attributed to GNN's efficient message passing mechanism to encode network topology and node content/features in a unified latent space, through iterative feature aggregation of neighborhoods for each node [3], [4]. Because node content (or node features) are propagated through network topology, both node features and topological structures are naturally preserved throughout the interactive learning process between features and structures. Under this learning paradigm, tremendous effort has been focused on developing effective feature aggregators for improved node representation learning, such as GAT [5] that learns different importance weights for various neighborhoods and

This work was supported in part by the US National Science Foundation (NSF) through Grants No. IIS-1763452 \& CNS-1828181.

M. Shi, Y. Tang, and X. Zhu are with the Department of Computer \& Electrical Engineering and Computer Science, Florida Atlantic University, Boca Raton, FL 33431 USA. E-mail: \{mshi2018, tangy, xzhu3\}@fau.edu.

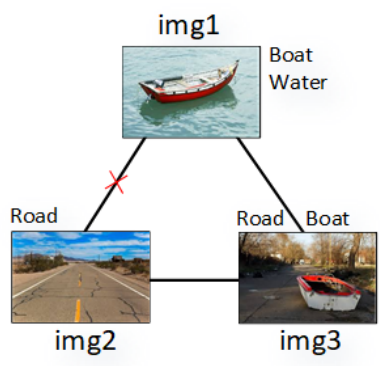

(a) Image network

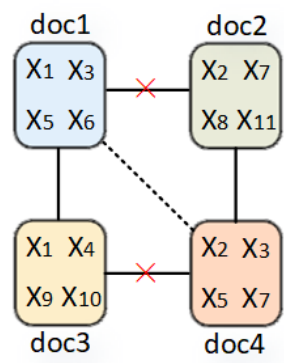

(b) Document network

Fig. 1. An illustration of inconsistent/incomplete relationships in networks. (a) an image network. (b) a document network. Topology of both networks are given but are not always consistent to the node content. For example, when using image semantics as node content, the edge between img 1 and img2 is inconsistent as they do not share any common semantics (they might be connected because of other unobserved correlations, such as pictures taken during the same trip). Analogically, the inconsistencies exist between (doc1, doc2) and (doc3, doc4), and there is a potential missing edge between doc 1 and doc 4 due to common features $\mathrm{X}_{3}$ and $\mathrm{X}_{5}$.

GraphSAGE [4] that learns a set of aggregator functions for each node.

Despite of promising results, all existing graph convolutional learning approaches employ a topology-driven principle to "force" node content to be aggregated by following edge connections. By doing so, they consider that node features and edge relationships are largely consistent and are mutually enhanced during the learning [6]. In reality, network node content (e.g., text and image semantics) and graph topology (e.g., link relations) may be highly inconsistent due to unconscious or deliberate human behaviors [7], [8]. For example, irrelevant citations between scholarly publications result in inconsistent citation networks. Similarly, an attacker may create fake followers or manipulate friendships, resulting in inconsistent social networks [9]. In addition, incomplete or missing links between nodes are also common, i.e., an image graph based on tag sharing rules often have sparse tag information therefore results in missing edges. Fig. 1 shows two examples of inconsistent and incomplete graphs or networks. In summary, graph inconsistency combined with sparse node relations severely challenge existing GNN learning models for following two reasons:

- Noisy Message Passing: When neighborhood relation- 


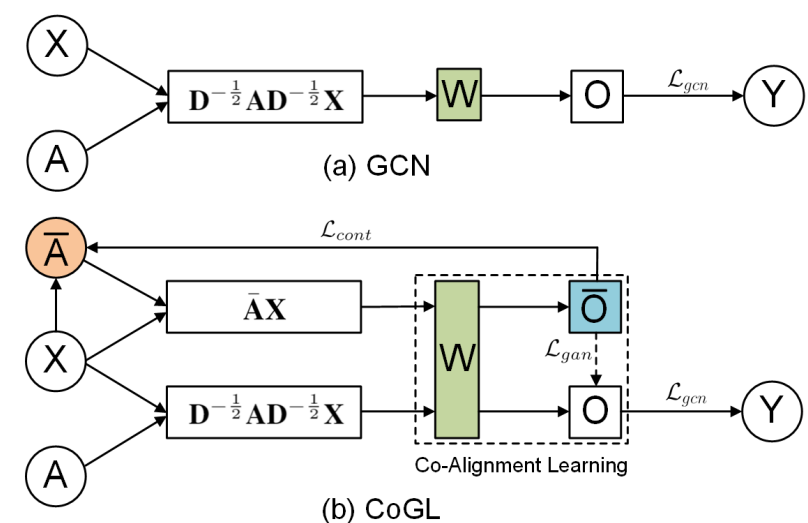

Fig. 2. The difference between GCN and our proposed CoGL. (a) GCN learns graph embeddings based on network topology $\mathbf{A}$ and node content $\mathbf{X}$ by optimizing classification loss $\mathcal{L}_{g c n}$. (b) CoGL first learns to construct a content network $\overline{\mathbf{A}}$ and then performs content and topology co-alignment embedding learning by optimizing a unified loss from three parts: content network construction loss $\mathcal{L}_{\text {cont }}$, node classification loss $\mathcal{L}_{\text {gcn }}$ and adversarial training loss $\mathcal{L}_{\text {gan }}$.

ships are misaligned to node affinities reflected by node content, nodes will aggregate irrelevant information from neighbors, resulting in noisy content and inferior representation learning. Such noisy messages will continue to pass through graph structures and finally deteriorate the learning of all nodes.

- Node Relationship Impairing: When two nodes have similar node content but there is no link between them, the missing link will "force" similar nodes to be dissimilar in embedding space. In addition, these absent relations will further impair the relation modeling between other pairs of nodes over the entire network.

Notice that network topology is often not optimal, a recent work [10] proposes to optimize network topology structure to improve graph convolutional network learning. However, such approach is potentially risky because revising network topology to satisfy optimization tends to overfit to training data. Alternatively, in this paper, we propose taking a different route to co-align network topology and node content for graph convolutional learning.

Specifically, we focus on information networks where nodes have rich features (content) such as texts and images. Instead of employing the traditional topology-driven principle, we treat network topology and node content as two distinct, but highly correlated, sources of data, and explicitly characterize their differences for embedding learning. We advocate a new "co-alignment" learning principle where the topology network should respect underlying node content, and the content should comply to the topology network for optimized representation learning. We propose a GNN model namely Co-alignment Graph convolutional Learning (CoGL) for this purpose.

To enable co-alignment learning, CoGL reconstructs a content network from node features. The content network together with the original topology network are then set to perform co-alignment learning in an adversarial fashion: (1) the content network aims to learn good embeddings complying to graph topology while (2) the topology network trains to learn optimal embeddings with shared learning parameters for semi-supervised node classification. In addition, the content network enforces adversarial training on the topology network in order to balance node content and topology information for optimal node representation learning. The difference between our proposed CoGL with existing Graph convolutional Networks (GCN) [3] is explained in Fig. 2

In summary, our main contribution is twofold: 1) We propose modeling inconsistency and discrepancy between network node content and topology for optimal and robust graph embedding; 2) We propose CoGL, a novel GNN model that enables co-alignment leaning between content based network and the original topology network.

\section{RELATED WORK}

Given a network with edge connections and content (features) associated to each node, graph embedding learns a low-dimensional vector for each node to preserve node content and network topology [11]. Many works have been proposed, ranging from unsupervised learning methods such as DeepWalk [12] to supervised learning methods such as SemiGraph [13]. The common idea is that nodes with similar topology or similar content should be represented with similar embeddings in the latent space [14].

Graph Neural Networks (GNN) [6] is a family of neural network models specifically developed for learning gridlike networked data. GNN models usually have efficient information aggregators [15] that apply directly on graphs to easily incorporate graph structures and features for unified node representation learning. Graph Convolutional Networks (GCN) [3] adopt a spectral-based convolution filter by which nodes can aggregate features from their respective local graph neighborhoods for representation learning. This convolution learning mechanism has been proven successful in many real-word analytic tasks such as link prediction [16], image recognition [2] and new drug discovery [17]. Following the similar convolution graph embedding scheme, many GNN models with more efficient information aggregators have been proposed. For example, Graph Attention Network (GAT) [5] learns to assign different importance weights for various nodes so that nodes can highlight important neighborhoods while aggregating features. GraphSAGE [4] learns a set of aggregation functions for each node to flexibly aggregate information from neighborhoods within different hops.

For all existing GNN approaches, graph convolutional learning is carried out by using topology to drive feature aggregation. A widely accepted assumption is that node content and graph structures are consistent and complementary for measuring node closeness in the embedding space [18], 


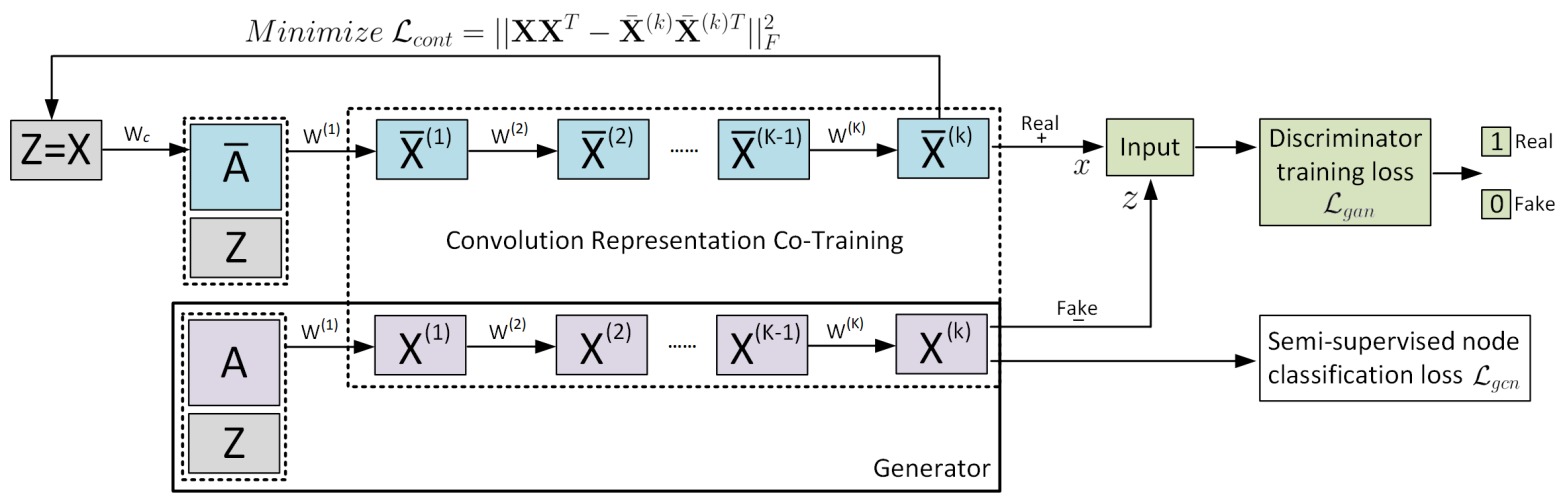

Fig. 3. The proposed CoGL model. It performs two convolution representation learning on content network $(\overline{\mathbf{A}})$ and topology network $(\mathbf{A})$, respectively. The content network tries to learn good embeddings that best reconstruct $\overline{\mathbf{A}}$ by minimizing $\mathcal{L}_{\text {cont }}$, while the topology network tries to learn optimal embeddigs with co-trained parameters by optimizing node classification loss $\mathcal{L}_{g c n}$. Meanwhile, The content network enforces an adversarial training on the topology network by optimizing binary classification loss $\mathcal{L}_{\text {gan }}$.

[19]. In reality, the network topology is often noisy and inconsistent to node content. A recent study [20] shows that network topology is not only inconsistent to node content at the individual node level, but is also different from the affinity network (built from node content) at the network level (e.g. degree distributions). A recent topology optimization based GCN [10] proposes to revise network topology to improve GCN learning, but requires revising/changing network topology which is risky to most users.

Different from existing models that consider graph structures and node content as consistent by default or revise them to ensure consistency, we propose a new co-alignment paradigm to explicitly model their inconsistency for optimal and robust graph embedding.

\section{Problem Definition AND Framework}

\section{A. Problem Definition}

Without loss of generality, an information graph can be represented as $\mathbf{G}=(\mathbf{V}, \mathbf{E}, \mathbf{X})$, where $\mathbf{V}=\left\{v_{i}\right\}_{i=1, \cdots,|\mathbf{V}|}$ is a set of unique nodes and $\mathbf{E}=\left\{e_{i, j}\right\}_{i, j=1, \cdots,|\mathbf{V}| ; i \neq j}$ is a set of edges which can be equal to a $|\mathbf{V}| \times|\mathbf{V}|$ adjacency matrix $\mathbf{A}$ with $\mathbf{A}_{i, j}=w_{i, j}>0$ if $e_{i, j} \in \mathbf{E}$ and $\mathbf{A}_{i, j}=0$ if $e_{i, j} \notin \mathbf{E} . \mathbf{X} \in \mathbb{R}^{|\mathbf{V}| \times m}$ is a matrix containing all $|\mathbf{V}|$ nodes with their features, i.e., $\mathbf{X}_{i} \in \mathbb{R}^{m}$ represents the feature vector of node $v_{i}$, where $m$ is the feature vector's dimension. It is easy to conclude that $\mathrm{G}$ can be any type of networked data where nodes have feature contents, such as citation networks with word texts as node features and image networks with image semantics as node features. Given an information graph $\mathrm{G}$, we aim to learn graph embeddings $\mathrm{O} \in \mathbb{R}^{|\mathbf{V}| \times c}$ for node classification in a semi-supervised fashion, where $c$ is the embedding size. In this paper, the expressions of graph embedding and representation learning are used interchangeably.

\section{B. The Overall Framework}

The proposed CoGL model for semi-supervised graph embedding and node classification is shown in Fig. 3. It performs a co-alignment learning between the constructed content network and the original topology network through three collaborative components:

- Content-aligned Graph Topology Learning: This part trains to learn the content-aligned graph topology network $\overline{\mathbf{A}}$ (content network) from input node features $\mathbf{X}$ by minimizing a graph reconstruction loss.

- Semi-supervised Graph Embedding: This part trains to learn convolution graph embeddings from the original graph topology network (topology network) A for node classification in a semi-supervised manner.

- Adversarial Graph Embedding Training: In this part, the content network embedding enforces an adversarial training on the topology network embedding.

\section{The Proposed Model}

\section{A. Content-aligned Graph Topology Learning}

We aim to learn a non-negative matrix $\overline{\mathbf{A}} \in \mathbb{R}^{|\mathbf{V}| \times|\mathbf{V}|}$ (e.g., we also call the content network) that could reveal the underlying pairwise node relationships from node features $\mathbf{X}$. An ad-hoc solution is to build a $k$-nearest neighbor graph [21] or simply calculate the Euclidean distance for each pair of nodes by $\overline{\mathbf{A}}_{i, j}=\left\|\mathbf{X}_{i}-\mathbf{X}_{j}\right\|$. However, this does not generate optimal graph embeddings for specific problems. Instead, we adopt a single-layer feed-forward neural network parametrized by a weight vector $\mathbf{W}_{c} \in \mathbb{R}^{m \times 1}$ and followed by a non-linear transformation (e.g., $\operatorname{ReLU}(u)=\max (0, u)$ ). It takes the feature difference $\left|\mathbf{X}_{i}-\mathbf{X}_{j}\right|$ between $i^{\text {th }}$ and $j^{t h}$ nodes as input and outputs the corresponding relevance weight by:

$$
w_{i, j}=\operatorname{ReLU}\left(\mathbf{W}_{c}^{T}\left|\mathbf{X}_{i}-\mathbf{X}_{j}\right|\right)
$$

Then, we apply softmax normalization on each node and finally obtain the weight matrix $\overline{\mathbf{A}}$ as: 


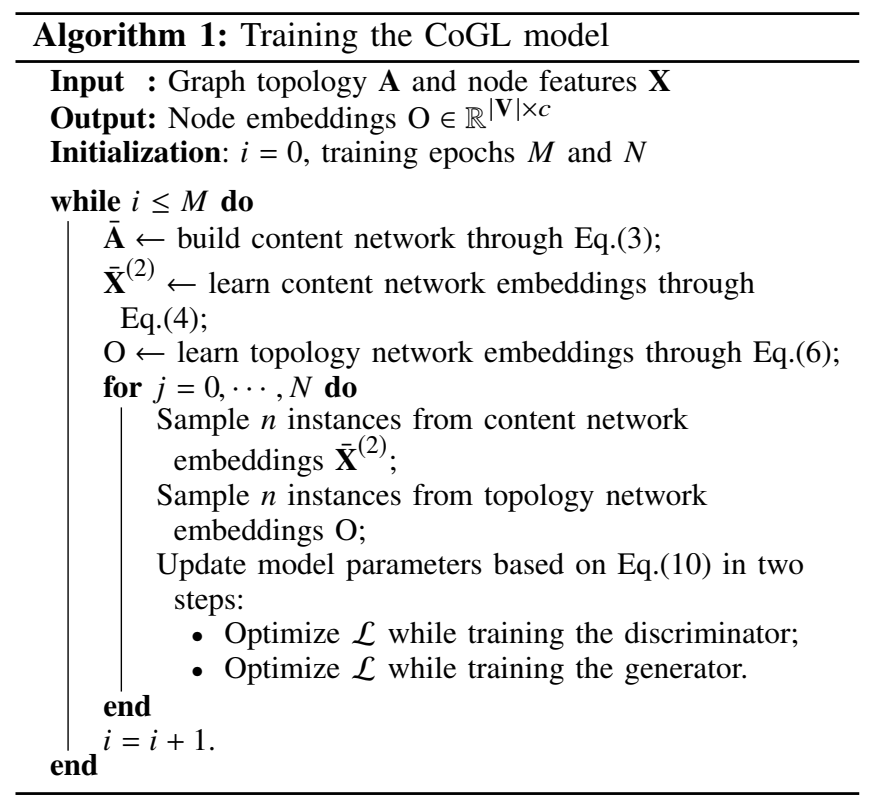

$$
\overline{\mathbf{A}}_{i, j}=\operatorname{softmax}\left(w_{i, j}\right)=\frac{\exp \left(\operatorname{ReLU}\left(\mathbf{W}_{c}^{T}\left|\mathbf{X}_{i}-\mathbf{X}_{j}\right|\right)\right)}{\sum_{j=1}^{|\mathbf{V}|} \exp \left(\operatorname{ReLU}\left(\mathbf{W}_{c}^{T}\left|\mathbf{X}_{i}-\mathbf{X}_{j}\right|\right)\right)}
$$

We can observe that $\overline{\mathbf{A}}$ is no longer a symmetric matrix (i.e., $\overline{\mathbf{A}}_{i, j} \neq \overline{\mathbf{A}}_{j, i}$ ) after the normalization, which is rational as the target is to learn an aligned approximation of nodes to their respective neighborhoods for optimal graph embedding. For efficient calculation, we can first map input node features (X) to a dimension-reduced space [5], [22] and Equation (2) can be written as:

$$
\overline{\mathbf{A}}_{i, j}=\frac{\exp \left(\operatorname{ReLU}\left(\mathbf{W}_{c}^{T}\left|\mathbf{X}_{i} \mathbf{W}_{p}-\mathbf{X}_{j} \mathbf{W}_{p}\right|\right)\right)}{\sum_{j=1}^{|\mathbf{V}|} \exp \left(\operatorname{ReLU}\left(\mathbf{W}_{c}^{T}\left|\mathbf{X}_{i} \mathbf{W}_{p}-\mathbf{X}_{j} \mathbf{W}_{p}\right|\right)\right)}
$$

where $\mathbf{W}_{p} \in \mathbb{R}^{m \times d}$ is a learned matrix and $d<m$.

To derive a consistent content network that best aligns node features, we first perform a two-layer convolutional representation learning [3] based on $\overline{\mathbf{A}}$ by:

$$
\overline{\mathbf{X}}^{(2)}=\overline{\mathbf{A}} \operatorname{ReLU}\left(\overline{\mathbf{A}} \mathbf{X} \mathbf{W}^{(1)}\right) \mathbf{W}^{(2)}
$$

where $\mathbf{W}^{(1)} \in \mathbb{R}^{m \times h}$ and $\mathbf{W}^{(2)} \in \mathbb{R}^{h \times c}$ are the first and second convolution-layer embedding parameters, respectively. Then, we minimize the reconstruction error between learned node embeddings and input node features as:

$$
\mathcal{L}_{\text {cont }}=\left\|\mathbf{X} \mathbf{X}^{T}-\overline{\mathbf{X}}^{(2)} \tilde{\mathbf{X}}^{(2) T}\right\|_{F}^{2}
$$

where both $\mathbf{X}$ and $\overline{\mathbf{X}}^{(2)}$ have been normalized to ensure stable parameter learning, i.e., through $\mathbf{X}=\operatorname{softmax}(\mathbf{X})$ and $\overline{\mathbf{X}}^{(2)}=$ $\operatorname{softmax}\left(\overline{\mathbf{X}}^{(2)}\right)$.
TABLE I

DOCUMENT NETWORK CHARACTERISTICS.

\begin{tabular}{c|c|c|c|c}
\hline Items & Cora & Citeseer & PubMed & DBLP \\
\hline \# Nodes & 2708 & 3327 & 19717 & 17725 \\
\hline \# Edges & 5429 & 4732 & 44338 & 52890 \\
\hline \# Features & 1433 & 3703 & 500 & 6974 \\
\hline \# Classes & 7 & 6 & 3 & 4 \\
\hline
\end{tabular}

TABLE II

IMAGE NETWORK CHARACTERISTICS.

\begin{tabular}{c|c|c|c|c}
\hline Items (\#) & Nodes & Edges & Features & Classes \\
\hline MIR & 5892 & 380808 & $500 \times 375$ & 152 \\
\hline ImageCLEF & 3461 & 221185 & $500 \times 375$ & 134 \\
\hline
\end{tabular}

\section{B. Semi-supervised Graph Embedding}

We perform graph representation learning for node classification in a semi-supervised manner. In a similar way, the low-dimensional embeddings are derived through a twolayer recursive convolution learning based on the original topology network A by:

$$
\mathrm{O}=\tilde{\mathbf{A}} \operatorname{ReLU}\left(\tilde{\mathbf{A}} \mathbf{X} \mathbf{W}^{(1)}\right) \mathbf{W}^{(2)}
$$

where $\tilde{\mathbf{A}}=\mathbf{D}^{-\frac{1}{2}}(\mathbf{I}+\mathbf{A}) \mathbf{D}^{-\frac{1}{2}}$ denotes the normalized form of $\mathbf{A}, \mathbf{I}$ is an identity matrix with the same shape and $\mathbf{D}$ is the degree matrix of $(\mathbf{I}+\mathbf{A})$. Here, the convolution learning parameters $\mathbf{W}^{(1)}$ and $\mathbf{W}^{(2)}$ are shared and co-trained between the content network and the topology network, which are beneficial to balance node content and topology for graph construction and embedding learning. Then, we do a semisupervised training to learn the parameters by minimizing the node classification loss $\mathcal{L}_{\text {gan }}$ as follows:

$$
\begin{gathered}
\mathrm{H}=\operatorname{softmax}(\mathrm{O})=\frac{\exp (\mathrm{O})}{\sum_{t} \exp \left(\mathrm{O}_{t}\right)} \\
\mathcal{L}_{g c n}=-\sum_{i \in \mathcal{Y}_{L}} \sum_{j=1}^{c} \mathrm{Y}_{i, j} \ln \mathrm{H}_{i, j}
\end{gathered}
$$

where the node embedding size $c$ equals the total number of labels for graph nodes. $\mathrm{Y} \in \mathbb{R}^{|\mathbf{V}| \times c}$ denotes the one-hot label indicators matrix for all nodes and $\mathcal{Y}_{L}$ is a set of node indices with labels known for semi-supervised training.

\section{Adversarial Graph Embedding Training}

The learning balance of content and topology is achieved through adversarial training [23], where the goal is to consider both content and topology information for optimal graph embedding and node classification. As shown in Fig. 3. the topology network learning component is considered a generator $\mathcal{G}(\mathbf{A}, \mathbf{X})$ that generates node embeddings based on the topology network A. During adversarial training, the discriminator tries to classify the real node (positive) sample $x \in \overline{\mathbf{X}}^{(2)}$ learned from the content network $\overline{\mathbf{A}}$ as class 1 and meanwhile classify the fake node (negative) sample $z=\mathcal{G}(\mathbf{A}, \mathbf{X})$ as class 0 . On the contrary, the generator tries 
TABLE III

Node Classification accuracy on Cora, Citeseer, Pubmed and DBLP datasets.

\begin{tabular}{c|cccc}
\hline Methods & Cora & Citeseer & Pubmed & DBLP \\
\hline DeepWalk & $67.2 \%$ & $43.2 \%$ & $65.3 \%$ & $66.3 \%$ \\
SemiEmd & $59.0 \%$ & $59.6 \%$ & $71.7 \%$ & $72.1 \%$ \\
Planetoid & $75.7 \%$ & $64.7 \%$ & $77.2 \%$ & $74.7 \%$ \\
Chebyshev & $81.2 \%$ & $69.8 \%$ & $74.4 \%$ & $74.3 \%$ \\
GCN & $81.5 \%$ & $70.9 \%$ & $79.0 \%$ & $75.1 \%$ \\
GAT & $83.2 \pm 0.7 \%$ & $71.0 \pm 0.7 \%$ & $79.0 \pm 0.3 \%$ & $78.2 \pm 0.7 \%$ \\
\hline CoGL (Ours) & $\mathbf{8 4 . 1} \pm 0.6 \%$ & $\mathbf{7 2 . 4} \pm 0.5 \%$ & $\mathbf{7 9 . 2} \pm 0.3 \%$ & $\mathbf{7 9 . 8} \pm 0.6 \%$ \\
\hline
\end{tabular}

to fool the discriminator by classifying $z$ as class 1 . The adversarial embedding training objective is given as:

$$
\begin{array}{r}
\min _{\mathcal{G}} \max _{\mathcal{D}} \mathcal{L}_{\text {gan }}(\mathcal{D}, \mathcal{G})=\mathbb{E}_{x \sim \overline{\mathbf{X}}^{(2)}} \log \mathcal{D}(x) \\
+\mathbb{E}_{z} \log (1-\mathcal{D}(\mathcal{G}(\mathbf{A}, \mathbf{X})))
\end{array}
$$

\section{Model Optimization and Training}

As described above, the content network construction and graph embedding conform to an adversarial co-alignment learning fashion for optimal node classification performance. As the three components in Fig. 3 share and co-train convolution graph embedding parameters $\mathbf{W}^{(1)}$ and $\mathbf{W}^{(2)}$, we finally seek to optimize the following combined objective as:

$$
\mathcal{L}=\mathcal{L}_{\text {gcn }}+\alpha \mathcal{L}_{\text {cont }}+\beta \mathcal{L}_{\text {gan }}(\mathcal{D}, \mathcal{G})
$$

where $\alpha$ and $\beta$ are set to balance the content network construction and the adversarial embedding training, respectively. Note that $\mathcal{L}_{\text {gan }}(\mathcal{D}, \mathcal{G})$ in Equation (9) involves both discriminator and generator training, where each part needs to combine both $\mathcal{L}_{g c n}$ and $\mathcal{L}_{\text {cont }}$ for collective model parameter optimization. The training procedure of our CoGL model is summarized in Algorithm 1.

\section{EXPERIMENTS}

In this section, we evaluate the proposed model for supervised node classification on six benchmark datasets. The dataset statistics are summarized in Tables 1 and 2.

\section{A. Datasets}

We use four document networks Cora, Citeseer, Pubmed and DBLP that have been widely used for node-level classification in previous work [3], [24]. Cora contains 2708 research papers grouped into 7 machine learning classes such as Reinforce Learning and Genetic Algorithms. There are 5429 edges between them and each paper node is described with a feature vector of 1433 dimensions. Citeseer contains 3327 research papers in 6 classes with 4732 links between them, where each paper node has a feature vector of 3703 dimensions. Pubmed contains 19717 literature nodes and
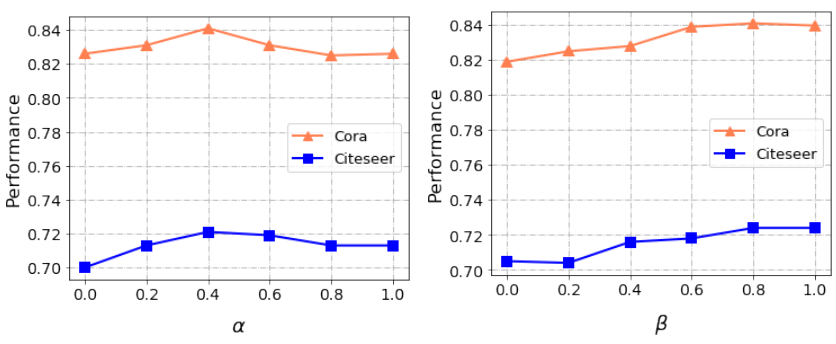

Fig. 4. Impact of parameters $\alpha$ and $\beta$.

44338 edges. Each node belongs to one of the 3 classes and has a feature vector of 500 dimensions. DBLP contains 17725 publications from 4 classes. It has 52890 edges and each node is associated with a feature vector of 6974 dimensions.

We also use two multi-label image networks 1 MIR and ImageCLEF. MIR has 5892 nodes from 152 classes. Each node represents a $500 \times 375 \mathrm{RGB}$ color image and there are 380808 edges for this network. ImageCLEF contains 3461 nodes and 221185 edges. Each node is also a $500 \times 375$ RGB image that corresponds to one or more of the 134 classes. For each image in these two datasets, we extract a CNN feature descriptor and the feature dimensions for MIR and ImageCLEF are transformed to 152 and 134, respectively.

\section{B. Compared Methods and Settings}

Compared Methods. We compare our proposed CoGL model for semi-supervised node classification against various state of the arts, including the random walk-based unsupervised embedding method (DeepWalk) [12], the semi-supervised embedding methods SemiEmb [25] and Planetoid [26], graph neural network models such as the spectrum-based graph convolutional networks (GCN) [3], the Chebyshev [27] filter-based GCN and the graph attention networks (GAT) [5] that additionally take importance of neighborhoods into consideration.

Experimental Settings. For experiments of baseline methods on Cora, Citeseer, Pubmed and DBLP datasets, we

\footnotetext{
${ }^{1}$ https://snap.stanford.edu/data/web-flickr.html
} 
TABLE IV

NODE CLASSIFICATION ACCURACY (\%) ON MIR AND IMAGECLEF DATASETS.

\begin{tabular}{c|ccc|ccc}
\hline Datasets & \multicolumn{3}{c|}{ MIR } & \multicolumn{3}{c}{ ImageCLEF } \\
\hline \# Labeled Nodes & 300 & 500 & 700 & 300 & 500 & 700 \\
\hline DeepWalk & $41.42 \pm 0.22$ & $42.98 \pm 0.25$ & $44.50 \pm 0.33$ & $43.34 \pm 0.50$ & $43.67 \pm 0.39$ & $44.98 \pm 0.43$ \\
SemiEmd & $46.75 \pm 0.39$ & $47.24 \pm 0.48$ & $48.61 \pm 0.27$ & $54.30 \pm 0.67$ & $55.22 \pm 0.49$ & $56.50 \pm 0.42$ \\
Planetoid & $50.74 \pm 0.33$ & $51.04 \pm 0.40$ & $51.91 \pm 0.23$ & $56.75 \pm 0.36$ & $57.77 \pm 0.36$ & $57.99 \pm 0.49$ \\
Chebyshev & $53.28 \pm 0.32$ & $54.28 \pm 0.32$ & $55.87 \pm 0.45$ & $58.12 \pm 0.37$ & $58.79 \pm 0.51$ & $59.58 \pm 0.51$ \\
GCN & $55.43 \pm 0.41$ & $56.14 \pm 0.38$ & $57.65 \pm 0.27$ & $60.33 \pm 0.34$ & $61.04 \pm 0.54$ & $61.60 \pm 0.38$ \\
GAT & $55.59 \pm 0.54$ & $57.27 \pm 0.76$ & $58.38 \pm 0.48$ & $61.45 \pm 0.52$ & $61.47 \pm 0.32$ & $62.32 \pm 0.41$ \\
\hline CoGL $($ Ours $)$ & $\mathbf{5 7 . 2 3} \pm \mathbf{0 . 4 6}$ & $\mathbf{5 8 . 7 8} \pm \mathbf{0 . 3 9}$ & $\mathbf{6 0 . 1 1} \pm \mathbf{0 . 4 5}$ & $\mathbf{6 1 . 7 7} \pm \mathbf{0 . 5 7}$ & $\mathbf{6 2 . 5 2} \pm \mathbf{0 . 4 0}$ & $\mathbf{6 3 . 8 6} \pm \mathbf{0 . 5 2}$ \\
\hline
\end{tabular}
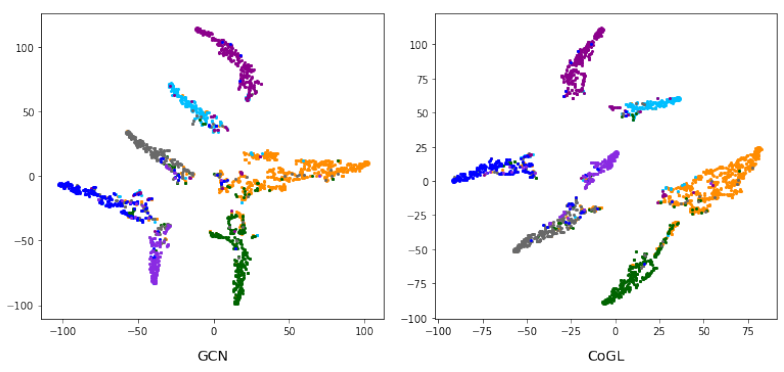

Fig. 5. Embedding 2-D visualization on Cora dataset.

follow the same settings as in previous works [3], [5]. For the MIR and ImageCLEF datasets, we respectively select 300, 500 and 700 labeled image nodes for training the model. For other unlabeled nodes, we select 1000 and 2000 images for validation and test, respectively. For each experiment, data splits are performed randomly and the average performances are finally reported over ten runs.

In our approach, the hidden layer dimensions $d$ for content network construction is set as 70 and the hidden convolution layer dimension $h$ is set as 30. We train CoGL for a maximum of 1000 epochs based on the Adam algorithm with an early stopping of 200 epochs. For the four document networks, the dropout probability and learning rate are set as 0.5 and 0.002 , and they are 0.2 and 0.01 for MIR and ImageCLEF datasets. For comparison, we set the balance parameters $\alpha$ and $\beta$ as 0.4 and 0.8 , respectively. Similar to previous work [3], we use a $L_{2}$ norm regularization where the weight decay is set as $5 \mathrm{e}-4$.

\section{Node Classification Results}

Table 3 shows the node classification results on Cora, Citeseer, Pubmed and DBLP datasets, and the results for MIR and ImageCLEF datasets are shown in Table 4. The best results in the two tables are highlighted. We can observe that our CoGL model outperforms other GNN models such as GCN and GAT for semi-supervised node classification. Existing GNN models naturally consider network structures and node features as two unbiased sources of data where

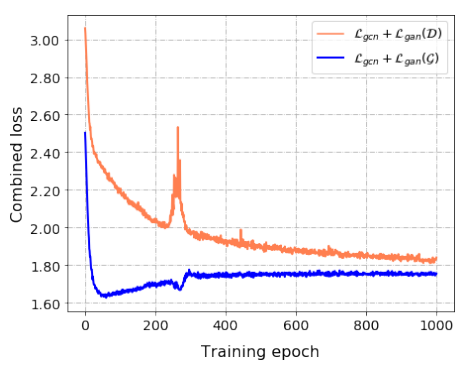

Fig. 6. Classification training loss.

each part consistently reveals and enhances the another during learning. However, the topology-based node relations are not always matched with the content-based node relations due to the existence of irrelevant and missing links between nodes in the original graph. Our method can model this discrepancy with the co-alignment learning between the content and topology networks. The experimental results of CoGL compared with existing GNN models and other state-of-theart baselines such as SemiEmd and DeepWalk demonstrates the effectiveness of our approach for information network learning. In addition, the visualization results in Fig. 5 show that CoGL can learn more discriminative node embeddings compared with GCN.

\section{Parameter Sensitivity and Classification Loss}

The impacts of parameters $\alpha$ and $\beta$ are shown in Fig. 4. We observe that either ignoring the content network construction component ( $\alpha=0.0$ ) or ignoring the adversarial training component $(\beta=0.0)$ during training causes the lowest classification performance, which verifies the benefit of combining content network and topology network for coalignment training in this paper.

To demonstrate the balanced learning between topology network and content network, we show the combined loss of node classification $\left(\mathcal{L}_{g c n}\right)$ and adversarial binary classification (discriminator $\mathcal{L}_{\text {gan }}(\mathcal{D})$ or generator $\mathcal{L}_{\text {gan }}(\mathcal{G})$ ) in Fig. 6. We observe that the two curves tend to be closer but there still a difference, which reflects an adversarial balance 
between topology network and content network for unified and optimal graph embedding and node classification.

\section{CONCLUSION}

Network topology and node content (including labels) are two main sources of information commonly used in many network embedding models. Although topology and node content are often inconsistent, existing methods, especially graph convolution networks, naturally ignore such inconsistency for embedding learning. In this paper, we took the inconsistency between graph structure and features into consideration for robust graph embedding learning, and proposed a novel co-alignment graph convolutional learning model, CoGL. The merit of CoGL lies in that it co-aligns the original topology network and the constructed content network for optimal node embedding and classification. Experiments and validations on six benchmark datasets demonstrated the effectiveness of CoGL. The proposed model can be used to learn embeddings for a variety of information networks with rich node content, such as the widely seen document networks and image networks.

\section{REFERENCES}

[1] W. Fan, Y. Ma, Q. Li, Y. He, E. Zhao, J. Tang, and D. Yin, "Graph neural networks for social recommendation," in The World Wide Web Conference. ACM, 2019, pp. 417-426.

[2] Z.-M. Chen, X.-S. Wei, P. Wang, and Y. Guo, "Multi-label image recognition with graph convolutional networks," in Proc. of IEEE CVPR, 2019, pp. 5177-5186.

[3] T. N. Kipf and M. Welling, "Semi-supervised classification with graph convolutional networks," arXiv preprint arXiv:1609.02907, 2016.

[4] W. Hamilton, Z. Ying, and J. Leskovec, "Inductive representation learning on large graphs," in Advances in Neural Information Processing Systems, 2017, pp. 1024-1034.

[5] P. Veličković, G. Cucurull, A. Casanova, A. Romero, P. Lio, and Y. Bengio, "Graph attention networks," arXiv preprint arXiv:1710.10903, 2017.

[6] Z. Wu, S. Pan, F. Chen, G. Long, C. Zhang, and P. S. Yu, "A comprehensive survey on graph neural networks," arXiv preprint arXiv:1901.00596, 2019.

[7] A. Hofmann, S. Perchani, J. Portisch, S. Hertling, and H. Paulheim, "Dbkwik: Towards knowledge graph creation from thousands of wikis." in International Semantic Web Conference (Posters, Demos \& Industry Tracks), 2017.

[8] H. Liu, L. Bai, X. Ma, W. Yu, and C. Xu, "Projfe: Prediction of fuzzy entity and relation for knowledge graph completion," Applied Soft Computing, vol. 81, p. 105525, 2019.

[9] A. Bojcheski and S. Günnemann, "Adversarial attacks on node embeddings," arXiv preprint arXiv:1809.01093, 2018.

[10] L. Yang, Z. Kang, X. Cao, D. Jin, B. Yang, and Y. Guo, "Topology optimization based graph convolutional network," in Proc. of the 28th IJCAI, 2019.

[11] M. Shi, Y. Tang, X. Zhu, J. Liu, and H. He, "Topical network embedding," Data Mining and Knowledge Disc., pp. 1-26, 2019.

[12] B. Perozzi, R. Al-Rfou, and S. Skiena, "Deepwalk: Online learning of social representations," in Proc. of ACM SIGKDD. ACM, 2014, pp. 701-710.

[13] R. Hisano, "Semi-supervised graph embedding approach to dynamic link prediction," in International Workshop on Complex Networks. Springer, 2018, pp. 109-121.

[14] H. Cai, V. W. Zheng, and K. C.-C. Chang, "A comprehensive survey of graph embedding: Problems, techniques, and applications," IEEE Trans. on KDE, vol. 30, no. 9, pp. 1616-1637, 2018.
[15] S. S. Du, K. Hou, R. R. Salakhutdinov, B. Poczos, R. Wang, and K. Xu, "Graph neural tangent kernel: Fusing graph neural networks with graph kernels," in Advances in Neural Information Processing Systems, 2019, pp. 5724-5734.

[16] M. Zhang and Y. Chen, "Link prediction based on graph neural networks," in Advances in Neural Information Processing Systems, 2018, pp. 5165-5175.

[17] M. Sun, S. Zhao, C. Gilvary, O. Elemento, J. Zhou, and F. Wang, "Graph convolutional networks for computational drug development and discovery," Brief. in bioinformatics, 2019.

[18] S. Pan, J. Wu, X. Zhu, C. Zhang, and Y. Wang, "Tri-party deep network representation," Proc. of IJCAI, 2016.

[19] A. Maheshwari, A. Goyal, A. Kumar, M. K. Hanawal, and G. Ramakrishnan, "Representation learning on graphs by integrating content and structure information," in 2019 11th International Conference on Communication Systems \& Networks (COMSNETS). IEEE, 2019, pp. 88-94.

[20] T. Guo, S. Pan, X. Zhu, and C. Zhang, "Cfond: Consensus factorization for co-clustering networked data," IEEE trans. on KDE, 2019.

[21] M. Maier, U. V. Luxburg, and M. Hein, "Influence of graph construction on graph-based clustering measures," in Advances in neural information processing systems, 2009, pp. 1025-1032.

[22] B. Jiang, Z. Zhang, D. Lin, J. Tang, and B. Luo, "Semi-supervised learning with graph learning-convolutional networks," in Proc. of IEEE CVPR, 2019, pp. 11313-11320.

[23] I. Goodfellow, J. Pouget-Abadie, M. Mirza, B. Xu, D. Warde-Farley, S. Ozair, A. Courville, and Y. Bengio, "Generative adversarial nets," in Advances in neural information processing systems, 2014, pp. 2672 2680.

[24] J. Leskovec and A. Krevl, "Snap datasets: Stanford large network dataset collection," 2014.

[25] J. Weston, F. Ratle, H. Mobahi, and R. Collobert, "Deep learning via semi-supervised embedding," in Neural Networks: Tricks of the Trade. Springer, 2012, pp. 639-655.

[26] Z. Yang, W. W. Cohen, and R. Salakhutdinov, "Revisiting semi-supervised learning with graph embeddings," arXiv preprint arXiv:1603.08861, 2016.

[27] M. Defferrard, X. Bresson, and P. Vandergheynst, "Convolutional neural networks on graphs with fast localized spectral filtering," in Advances in neural information processing systems, 2016, pp. 3844 3852 . 